\title{
On the Numerical Approximation of Unstable Minimal Surfaces with Polygonal Boundaries
}

\author{
M. Hinze
}

Sfb 288 Preprint No. 139

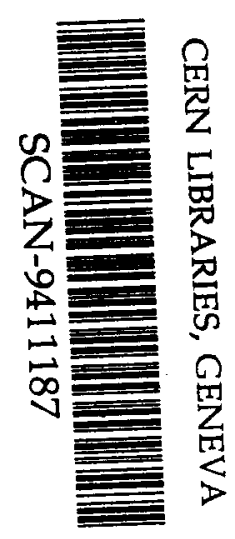

sw9446

Diese Arbeit ist mit Unterstützung des von der Deutschen Forschungsgemeinschaft getragenen Sonderforschungsbereiches 288 entstanden und als Manuskript vervielfältigt worden.

Berlin, Okt. 1994 


\title{
On the Numerical Approximation of Unstable Minimal Surfaces with Polygonal Boundaries
}

\author{
Michael Hinze \\ Fachbereich Mathematik \\ Technische Universität Berlin
}

\begin{abstract}
This work is concerned with the approximation and the numerical computation of polygonal minimal surfaces in $\mathbb{R}^{q}(q \geq 2)$. Polygonal minimal surfaces correspond to the critical points of Shiffman's function $\Theta$. Since this function is analytic, polygonal minimal surfaces can be characterized by means of the second derivative of $\Theta$. We present a finite element approximation of cuasiminimal surfaces together with an error estimate. In this way we obtain discrete approximations $\Theta_{h}$ of $\Theta$ and $f_{h}$ of $\nabla \Theta$. In particular we prove that the discrete functions converge uniformly on certain compact subsets. This will be the main tool for proving existence and convergence of discrete minimal surfaces in neighbourhoods of non-degenerate minimal surfaces. In the numerical part of this paper we compute numerical approximations of polygonal minimal surfaces by use of Newton's method applied to $f_{h}$.
\end{abstract}

\section{Introduction}

Let $\Gamma$ be a Jordan curve in $\mathbb{R}^{q}(q \geq 2)$ and denote by $B=\left\{w=\left(x_{1}, x_{2}\right) \in \mathbb{R}^{2}:|u|<1\right\}$ the unit disc in $\mathbb{R}^{2}$. A mapping $u \in C^{0}\left(\bar{B}, \mathbb{R}^{q}\right) \cap C^{2}\left(B, \mathbb{R}^{q}\right)$ is called disc type minimal surface spanning $\Gamma$ if $u$ satisfies

$$
\begin{aligned}
& \Delta u=0 \text { in } B \\
& \left|u_{x_{1}}\right|^{2}-\left|u_{x_{2}}\right|^{2}=\left(u_{x_{1}}, u_{x_{2}}\right)=0 \text { in } B \\
& u_{\mid B} \quad \text { is a (weakly) monotone parametrization of I'. }
\end{aligned}
$$

In the case where $\Gamma$ is a simple, closed polygon, the minimal surface problen is connected with the critical points of a function of finitely many variables. To be more precise, let $a_{1}, \ldots, a_{N+3}$ denote the distinct vertices of the polygon $\Gamma(N \geq 1)$ and consider mappings $v: \bar{B} \rightarrow \mathbb{R}^{q}$ in $C^{\prime 0}\left(\bar{B}, \mathbb{R}^{q}\right) \cap C^{1}\left(B, \mathbb{R}^{q}\right)$ such that $v(-1)=a_{N+1}, v(-i)=a_{N+2}, v(1)=a_{N+3}$ and $v\left(\epsilon^{i \kappa_{j}}\right)=a_{j}(1 \leq j \leq N)$, where $k=$ $\left(\kappa_{1}, \ldots, \kappa_{N}\right)$ is an $\mathrm{N}$-tuple of parameter values $\kappa_{j}$ satisfying $0<\kappa_{1}<\ldots<\kappa_{N}<\pi$. Let $F^{\prime}(\kappa)$ denote the class of such mappings which map the $\operatorname{arc} \bar{\gamma}_{j}:=\left\{e^{i \phi} ; \kappa_{j} \leq \phi \leq \kappa_{j+1}\right\}$ in a weakly monotone way onto the segment $\left[a_{j}, a_{j+1}\right]$ on the straight line $a_{j}+\Gamma_{j}$ through the points $a_{j}, a_{j+1}\left(\kappa_{j} \equiv \kappa_{i}\right.$ if $j \equiv$ $l \bmod N+3)(1 \leq j \leq N+2)$. Disc type minimal surfaces spanning $\Gamma$ now correspond in a one-to-one manner to the critical points of Courant's function

$$
d(\kappa):=\inf _{v \in F^{\prime}(\kappa)} D(v),
$$

where Dirichlet's integral is defined by

$$
D(v):=\frac{1}{2} \int_{B}|\nabla v|^{2} d x
$$


Problem (4) has a unique solution $v(\kappa)$, called minimal vector (see [2, Lemma 6.3]). The function $d$ is known to be of class $C^{1}$. Unfortunately, it is not known up to now whether $d$ is of class $C^{2}$, therefore Courant's function is not a good candidate to develop a Morse theory for polygonal minimal surfaces.

The Marx-Shiffman function $\Theta$ was introduced in the paper of Marx [16], which was based on a joint work of Marx and Shiffman concerning polygonally bounded minimal surfaces. Marx and Shiffman enlarged the class $F^{\prime}(\kappa)$ to the class $F(\kappa)$ of mappings which map the arc $\gamma_{j}$ into the straight lines $a_{j}+\Gamma_{j}$ and defined

$$
\Theta(\kappa):=\inf _{\nu \in F(\kappa)} D(v)
$$

The unique solution $u(\kappa)$ of problem (6) is called (normalized) quasiminimal surface. As a result, we clearly have $d(\kappa) \geq \Theta(\kappa)$ and $d(\kappa)>\Theta(\kappa)$ for certain values of $\kappa$ (see [15]). In [16], it was stated that $\Theta$ is of class $C^{\infty}$. In [5], Heinz proved that the quasiminimal surfaces $u(\kappa)$ depend analytically on $\kappa$. Using this result Heinz was able to prove that $\Theta$ is analytic ([6]). Furthermore, according to Heinz the critical points of $\Theta$ correspond in a one-to-one manner to solutions of a generalized Plateau problem for $\Gamma$. Here, generalized means : the solution $u(\kappa)$ can overshoot the vertices and we only know that $u\left(\gamma_{j}\right) \subset a_{j}+\Gamma_{j}$. Note, that $d$ and $\Theta$ are closely connected as they coincide at the critical points of $d$ (see [19]).

Sauvigny computed the Morse index of such generalized solutions by studying the second derivative of $\Theta$ in [20]. In this context it would be desirable to determine classes of polygons for which $d$ and $\Theta$ possess only a finite number of critical points. For arbitrary polygons this question is still open. But for a special class of extreme polygons Sauvigny completely investigated this question for the function $d$ ([19, Eindeutigkeitssatz]). Uniqueness and finiteness results for the set of critical points of the function $\Theta(\kappa)$ have not yet been obtained.

In the last years several numerical approaches to the boundary value problem for parametric minimal surfaces had been made. Here, we mention the works of Jarausch [14], Wohlrab [24], Tsuchija [21], [22],[23], Dziuk [3], Hutchinson [13], Pinkall/Polthier [18], Dziuk/Hutchinson [4] and refer to the references therein.

In this work we apply a new method to approximate minimal surfaces with polygonal boundaries. The method is based on the numerical treatment of $\Theta$ and a suitable approximation of $\nabla \Theta$. Using piecewise linear finite elements, we compute discrete quasiminimal surfaces $u_{h}(\kappa)$. In this way we obtain an approximation $\Theta_{h}(\kappa)=D_{h}\left(u_{h}(\kappa)\right)$ of $\Theta$. Based on an explicit expression for $\Gamma \Theta$, that only involves the quasiminimal surface $u(\kappa)$, we introduce a discrete approximation $f_{h}$ of $\nabla \Theta$. Applying Newton's method to the equation $f_{h}(\kappa)=0$ we then can compute approximations of critical points of $\Theta$. The corresponding quasiminimal surfaces in certain cases are approximations of generalized minimal surfaces. Since we can compute the eigenvalues of a finite difference approximation of $D^{2} \Theta_{h}$, we are able to classify these discrete minimal surfaces.

Our main result can be formulated as follows. Let $\kappa$ correspond to a non-degenerate critical point of $\Theta$ and denote by $u=u(\kappa)$ the associated minimal surface (which might be unstable). Then there are discrete minimal surfaces $u_{h}=u_{h}\left(\kappa_{h}\right)$ satisfying

$$
\left\|u-u_{h}\right\|_{1, B_{h}} \leq c h^{\mu^{2}}
$$

and

$$
\left|D(u)-D_{h}\left(u_{h}\right)\right| \leq c h^{2 \mu}
$$

where $\mu \pi$ denotes the smallest angle at the polygon vertices the minimal surface maps into and $h_{h}$ are roots of the function $f_{h}$ contained in some neighbourhood of $k$. 
This is the first convergence result for unstable minimal surfaces in non-smooth contours. In [4] Dziuk/Hutchinson applied a linear boundary element method to compute approximations of non-degenerate minimal surfaces that are bounded by smooth curves $\Gamma \subset \mathbb{R}^{q}$ and proved existence and convergence of discrete minimal surfaces in neighbourhoods of non-degenerate minimal surfaces. The first convergence for stable minimal surfaces can be found in Tsuchija's papers [22], [23].

For the visualization of grids and discrete surfaces we used the the graphical programing environment GRAPE developed at the Sonderforschungsbereich 256 at the university of Bonn, Germany.

\section{Notations and analytical preliminaries}

In what follows, $N$ and $q$ denote integers with $N \geq 1$ and $q \geq 2$. Let $B:=\left\{u:=x_{1}+i x_{2} ;|u|<1\right\} \subset$ $\mathbb{C} \equiv\left\{\mathrm{x}=\left(\mathrm{x}_{1}, \mathrm{x}_{2}\right) ;|\mathrm{x}|<1\right\} \subset \mathbb{R}^{2}$ denote the unit disc and let $\Gamma \subset \mathbb{R}^{q}$ be a closed polygon with $N+3$ distinct vertices $a_{1}, \ldots, a_{N+3}$ arranged in cyclical order. We set

$$
\begin{gathered}
\varphi_{k}:=\frac{a_{k+1}-a_{k}}{\left|a_{k+1}-a_{k}\right|} \quad\left(1 \leq k \leq N+3 ; a_{N+4}:=a_{1}\right) . \\
\Gamma_{k}=\left\{x \in \mathbb{R}^{q} ; x=t \varphi_{k}(\alpha), t \in \mathbb{R}\right\} \quad(1 \leq k \leq N+3)
\end{gathered}
$$

and denote by $S_{k}, T_{k}$ denote the reflections at the lines $\Gamma_{k}$ and $a_{k}+\mathrm{I}_{k}$, respectively. Let $k=$ $\left(\kappa_{1}, \ldots, \kappa_{N}\right) \in \mathbb{R}^{N}$ denote an $N$-tuple of parameter values $\kappa_{j}$ satisfying

$$
0<\kappa_{1}<\ldots<\kappa_{N}<\pi
$$

and define

$$
T:=\left\{\kappa \in \mathbb{R}^{N} ; 0<\kappa_{1}<\ldots<\kappa_{N}<\pi\right\} .
$$

In order to connect problem (6) with the Plateau problem for the polygon $\Gamma$ we sct

$$
\kappa_{N+1}:=\pi, \quad \kappa_{N+2}:=\frac{3 \pi}{2}, \quad \kappa_{N+3}:=2 \pi .
$$

$T^{\prime} \subset \subset T$ always denotes a compact subset of $T$. Furthermore, for $\kappa \in T$ we set

$$
\gamma_{k}(\kappa):=\left\{w=e^{i \phi} ; \kappa_{k}<\phi<\kappa_{k+1}\right\} \quad\left(1 \leq k \leq N+3 ; \kappa_{N+4} \equiv \kappa_{1}+2 \pi\right)
$$

and abbreviate

$$
\beta_{k}(\kappa):=e^{i \kappa_{k}} \quad(1 \leq k \leq N+3)
$$

For $m \in \mathbb{N}_{0}$ and $1 \leq p \leq \infty, H^{m, p}(\Omega)$ denotes the Sobolev space of all Lebesgue-measurable functions having $p$-integrable derivatives up to order $m$ equipped with the usual norm $\|\cdot\|_{m, p^{\prime}, \Omega}$. The corresponding seminorms are denoted by $|\cdot|_{m, p, \Omega}$. We abbreviate $H^{m}(\Omega) \equiv H^{m, 2}(\Omega),\|f\|_{m, \Omega} \equiv\|f\|_{m, 2, \Omega}$, $|f|_{m, \Omega} \equiv|f|_{m, 2, \Omega}$ as well as $\|f\|_{\Omega} \equiv\|f\|_{0, \Omega}$.

For $k \in \mathbb{N}_{0}, C^{k}(\Omega)$ denotes the space of all functions having continuous derivatives up to order $k$. With $C^{k}(\bar{\Omega})$ we denote the set of all functions in $C^{k}(\Omega)$, such that. for $|s| \leq k$, there exists a continuous extension of $\partial^{s} f$ onto $\bar{\Omega}$. 
$H^{m, p}\left(\Omega, \mathbb{R}^{q}\right)$ is defined as the set of all vector-valued functions $f=\left(f_{1}, \ldots, f_{q}\right)$, such that $f_{i} \in$ $H^{m, p}(\Omega)(1 \leq i \leq q)$. The function spaces $C^{k}\left(\Omega, \mathbb{R}^{q}\right)$ and $C^{k}\left(\bar{\Omega}, \mathbb{R}^{q}\right)$ are defined analogously.

Introducing the function spaces

$$
X(\kappa):=\left\{v \in H^{1}\left(B, \mathbb{R}^{q}\right) ; v\left(\gamma_{k}(\kappa)\right) \subset a_{k}+\Gamma_{k}(1 \leq k \leq N+3)\right\}
$$

and

$$
X_{0}(\kappa, \alpha):=\left\{v \in H^{1}\left(B, \mathbb{R}^{q}\right) ; v\left(\gamma_{k}(\alpha)\right) \subset \Gamma_{k}(\alpha) \quad(1 \leq k \leq N+3)\right\}
$$

problem (6) according to [11, Theorem 3.1] alternatively can be written as

$$
\Theta(\kappa)=\inf _{v \in X(\kappa)} D(v) \quad(\kappa \in T)
$$

This problem has a unique solution $u=u(\kappa) \in C^{0}\left(\bar{B}, \mathbb{R}^{q}\right) \cap C^{2}\left(\bar{B} \backslash\left\{\beta_{1}, \ldots, \beta_{N^{\prime}+3}\right\}\right.$, $\left.\mathbb{R}^{q}\right)$ that satisfies

$$
\int_{B} \nabla u \nabla v d x=0 \quad \forall v \in \mathrm{X}_{0}
$$

and solves the following system of partial differential equations (see [5, Satz 1])

$$
\begin{aligned}
\Delta u & =0 \quad \text { in } B \\
u\left(\gamma_{k}\right) & \subset a_{k}+\Gamma_{k} \quad 1 \leq k \leq N+3 \\
\left(\partial_{r} u, \varphi_{k}\right) & =0 \quad \text { on } \quad \gamma_{k}, \quad 1 \leq k \leq N+3 .
\end{aligned}
$$

Since $u$ is continuous on $\bar{B}$ we by necessity have

$$
u\left(\beta_{k}\right)=a_{k} \quad(k=1, \ldots N+3)
$$

At the vertices of $\Gamma$ the normal to the contour has a jump discontinuity. This causes a lack of regularity of $u$ at $\beta_{k}$. Heinz completely investigated this situation in [5, Satz 2]. In order to formulate the corresponding theorem we first introduce some notations. Let $\kappa^{*} \in T$ be arbitrary, but fixed. For $\epsilon>0$ we set

$$
U_{\epsilon}\left(\kappa^{*}, \beta_{k}\left(\kappa^{*}\right)\right):=\left\{(\kappa, w) \in \mathbb{C}^{N} \times \mathbb{C} ;\left|\kappa-\kappa^{*}\right|<\epsilon,\left|w-\beta_{k}\left(\kappa^{*}\right)\right|<\epsilon\right\} \quad(1 \leq k \leq N+3)
$$

and $\partial_{w}:=\frac{1}{2}\left(\partial_{x_{1}}-i \partial_{x_{2}}\right)\left(w=x_{1}+i x_{2}\right)$. Now we extract from [5, Satz 2$]$

Theorem 2.1 Let $D=D\left(\kappa^{*}\right) \subset \mathbb{C}$ denote a simply ronnected ifgion, such that $D \cap B$ is nonempty, connected and the closure $\bar{D}$ does not contain any of the points $\beta_{j}\left(\kappa^{*}\right)(j=1, \ldots N+3)$. Then. there exists a polydisc

$$
U_{D}\left(\kappa^{*}\right)=\left\{\kappa \in \mathbb{C}^{N} ;\left|\kappa_{\mathrm{j}}-\kappa_{\mathrm{j}}^{*}\right|<\epsilon_{\mathrm{D}}, \mathrm{j}=1 \ldots, \mathrm{N}\right\}
$$

and a function $f_{D}(\kappa, w)$, which is analytic in $U_{D}\left(\kappa^{*}\right) \times D$, such that the solution $u(\kappa)$ of $(16)$ satisfies

$$
u_{w}(\kappa)(w)=f_{D}(\kappa, w) \quad \forall(\kappa, w) \in\left(U_{D}\left(\kappa^{*}\right) \cap T\right) \times(D \cap B) .
$$

Furthermore, for every $k=1, \ldots, N+3$ there exists an integer $1 \leq p_{k} \leq q, p_{k}$ txponents $-1<p_{1}^{k}<$ $\ldots<p_{p_{k}}^{k} \leq 0$ and an $\epsilon_{0}>0$, such that for the solutions $u(k)$ of $(10)$ the following singular expansions are valid

$$
u_{w}(\kappa)(w)=\sum_{j=1}^{p_{k}} f_{j}^{k}(\kappa, w)\left(w-\beta_{k}(\kappa)\right)^{p_{j}^{k}} \quad \forall(\kappa, w) \in U_{\epsilon_{1}}\left(\kappa^{*}, \beta_{k}\left(\kappa^{*}\right)\right) \cap(T \times B) .
$$

The complex-valued coefficient functions $f_{j}^{k}(\kappa, w)$ are analytir in $l_{+\ldots}\left(\kappa^{*}, \beta_{k}\left(\kappa^{*}\right)\right)$. 
Integration of $(22)$ yields for some suitable $\tilde{\epsilon}_{0}>0$

$$
u(\kappa)(w)=a_{k}+\operatorname{Re}\left(\sum_{j=1}^{p_{k}} g_{j}^{k}(\kappa, w)\left(w-\beta_{k}(\kappa)\right)^{p_{j}^{k}+1}\right) \quad \forall(\kappa, w) \in U_{\tilde{\epsilon}_{0}}\left(\kappa^{*}, \beta_{k}\left(\kappa^{*}\right)\right) \cap(T \times B)
$$

with suitable coefficient functions $g_{j}^{k}(\kappa, w)$. The exponents $p_{j}^{k}$ are directly related to the eigenvalues of $S_{k-1} S_{k}^{-1}$ and therefore to the angles of the polygon $\Gamma$ at $a_{k}(1 \leq k \leq N+3)($ see $[5,(3.26),(3.28),(3.49),(3.63)])$. In the case $q=3$ we have from $[7,(4.2)]$

$$
p_{3}^{k}=0, \quad p_{2}^{k}=-\alpha_{k}, \quad p_{1}^{k}=\alpha_{k}-1 \quad(1 \leq k \leq N+3),
$$

where

$$
\cos \alpha_{k} \pi:=\varphi_{k-1}^{t} \varphi_{k} \quad(k=2, \ldots, N+3) .
$$

Using the exponents of Theorem 2.1 set

$$
p_{\min }:=\min _{1 \leq k \leq N+3} \min _{1 \leq j \leq p_{k}} p_{j}^{k} \in(-1,0] .
$$

Theorem 2.1 then implies for a suitable $\tilde{\epsilon}>0$ (compare also $[8,(2.1)]$ )

$$
\left|u_{w}(\kappa)(w)\right| \leq c \sum_{k=1}^{N+3}\left|w-\beta_{k}(\kappa)\right|^{p_{m i n}} \quad\left(|w|<1 ;\left|\kappa-\kappa^{*}\right|<\bar{l}\right)
$$

and

$$
\left|u_{w w}(\kappa)(w)\right| \leq c \sum_{k=1}^{N+3}\left|w-\beta_{k}(\kappa)\right|^{p_{m+n}-1} \quad\left(|w|<1 ;\left|\kappa-\kappa^{*}\right|<\hat{\epsilon}\right) .
$$

with some $c>0$.

Next, we define for $\epsilon>0$

$$
\Omega_{\epsilon}(\kappa):=\bar{B} \backslash \cup_{k=1}^{N+3} B_{\epsilon}\left(\beta_{k}(\kappa)\right), \quad \kappa \in T
$$

and assign to every $\kappa \in T$ a simply connected domain $D(\kappa)$ in such a way, that Theorem 2.1 is valid for $\kappa=\kappa^{*}$. Let $T^{\prime} \subset \subset T$ and apply Theorem 2.1 for every $\kappa \in T^{\prime}$ together with suitable domains $D(\kappa)$. Then the corresponding polydiscs in Theorem 2.1 induce an open covering of $T^{\prime}$. Thus, the Heine-Borel theorem together with $(27)$ and (28) proves

Corollary 2.1 For every $T^{\prime} \subset \subset T$ there exist positive constants $c_{1}\left(T^{\prime}\right), c_{2}\left(T^{\prime}, \epsilon\right)$ and $c_{3}\left(T^{\prime}\right)$ surh that

$$
\begin{gathered}
\|u(\kappa)\|_{1, p, B} \leq c_{1}\left(T^{\prime}\right) \quad \forall \kappa \in T^{\prime} \forall 2 \leq p<\frac{-2}{p_{\min }}, \\
\|u(\kappa)\|_{2, \Omega_{\epsilon}(\kappa) \leq c_{2}\left(T^{\prime}, \epsilon\right)} \forall \kappa \in T^{\prime} \forall \epsilon>0, \Omega_{\epsilon}(\kappa) \neq \emptyset
\end{gathered}
$$

and

$$
\left|u_{w w}(\kappa)(w)\right| \leq c_{3}\left(T^{\prime}\right) \sum_{k=1}^{N+3}\left|w-\beta_{k}(\kappa)\right|^{p_{m i n}-1} \quad \forall \kappa \in T^{\prime} \quad(|w|<1) .
$$

In particular for every $T^{\prime} \subset \subset T$, there exists some $\epsilon\left(T^{\prime}\right)>0$ so that the assertion in the second part of Theorem 2.1 is valid for every $\kappa=\kappa^{*} \in T^{\prime}$ and $\epsilon_{0}=\epsilon\left(T^{\prime}\right)$. For $\epsilon\left(T^{\prime}\right)$ inequality (31) reads

$$
\|u(\kappa)\|_{2, \Omega_{e\left(T^{\prime}\right)}(\kappa)} \leq c_{2}\left(T^{\prime}\right) \quad \forall \kappa \in T^{\prime}
$$

This Corollary will be in important tool in proving a uniform error estimate for discrete quasiminimal surfaces (see Section 3).

The next Theorem was proved by Heinz in $[6$, Satz $]$ and characterizes minimal surfaces in terms of $\Theta$. 
Theorem 2.2 For $\kappa \in T$ the surface $u(\kappa)$ is a minimal surface iff $\nabla \Theta(\kappa)=0$. $\Theta$ is analytic.

Consequently, Plateau's problem for $\Gamma$ is analytically embedded into a $N$-parameter family of quasiminimal surfaces.

In [12, Theorem 3.3.3] we computed the following expression for the partial derivatives of $\Theta$ with respect to $\kappa_{j}(j=1, \ldots, N+3)$

$$
\begin{array}{r}
\Theta_{\kappa_{j}}(\kappa)=-\frac{1}{2}\left\{\frac{1}{\kappa_{j+1}-\kappa_{j}} \int_{S\left(\kappa_{j}, \kappa_{j+1}\right)}\left(u_{x_{1}}^{2}(\kappa)-u_{x_{2}}^{2}(\kappa)\right) \frac{x_{1}^{2}-x_{2}^{2}}{x_{1}^{2}+x_{2}^{2}}+4 u_{x_{1}}(\kappa) u_{x_{2}}(\kappa) \frac{x_{1} x_{2}}{x_{1}^{2}+x_{2}^{2}} d x_{1} d x_{2}-\right. \\
\left.\frac{1}{\kappa_{j}-\kappa_{j-1}} \int_{S\left(\kappa_{j-1}, \kappa_{j}\right)}\left(u_{x_{1}}^{2}(\kappa)-u_{x_{2}}^{2}(\kappa)\right) \frac{x_{1}^{2}-x_{2}^{2}}{x_{1}^{2}+x_{2}^{2}}+4 u_{x_{1}}(\kappa) u_{x_{2}}(\kappa) \frac{x_{1} x_{2}}{x_{1}^{2}+x_{2}^{2}} d x_{1} d x_{2}\right\},
\end{array}
$$

where $S\left(\kappa_{j-1}, \kappa_{j}\right)$ denotes the sector of $B$ defined by the points $0, \beta_{j-1}, \beta_{j}(j=2, \ldots, N+3)$. Due to results of Heinz ([9, Satz 2]) and Sauvigny ([20, Theorem II]) the following Definition makes sense.

Definition 2.1 A minimal surface $u(\kappa)$ is called non-degenerate iff rank $D^{2} \Theta(\kappa)=. N$.

Lemma 2.1 Non-degenerate minimal surfaces are isolated.

Proof : Let $u(\kappa)$ be a non-degenerate minimal surfaces, i.e. $\nabla \Theta(\kappa)=0, \operatorname{rank} l^{2} \Theta(\kappa)=N$. By use of the Inverse-function theorem we obtain an open neighbourhood $V(\kappa)$ so that $\nabla \Theta(\bar{\kappa}) \neq 0, \tilde{\kappa} \in V(\kappa) \backslash\{\kappa\}$. This proves the Lemma.

We close this section with stating a Poincaré inequality for functions in

$$
V:=\left\{v \in H^{1}\left(B, \mathbb{R}^{q}\right) ; v\left(\gamma_{1}\right) \subset \Gamma_{1}, v\left(\gamma_{N+3}\right) \subset \Gamma_{N+3}\right\}
$$

(compare also $[5,(2.12)])$.

\section{Lemma 2.2}

$$
\exists c>0 \forall v \in V:\|v\|_{B} \leq c|v|_{1, B} .
$$

This lemma can be proved as Hilfsatz 2.6 in [10].

\section{The discrete approach}

Let $\kappa \in T$ be fixed. To obtain a conforming finite element method approximating problem (16) we first construct a finite dimensional affine space $X_{h}=X_{h}(\kappa) \subseteq X^{*}=X(\kappa)$. For this purpose denote by $\tau=\tau(\kappa)$ triangulations of $B$ that are obtained by one of the following refinement strategies applied to macro-triangulations of the unit disc of the form as indicated in Fig. $1 . k \in \mathbb{N}$ denotes the refinement level.

S1 :

1. (a) $\tau_{0}:=\cup_{j=1}^{N+3} \Delta\left(0, e^{i \kappa_{j-1}}, e^{i \kappa_{j}}\right)\left(\kappa_{0} \equiv \kappa_{N+3}-2 \pi\right)$ (macro-triangulation, Fig. 1)

(b) Mark the edge $\left[e^{i \kappa_{1-1}}, e^{i \kappa_{j}}\right]$, called refinement edge, of the triangle $\Delta\left(0, \epsilon^{i \kappa_{i-1}}, e^{i \kappa_{j}}\right)(j=$ $\left.1, \ldots, N+3 ; \kappa_{0} \equiv \kappa_{N+3}-2 \pi\right)$

(c) $l=0$

2. Bisect each $\Delta \in \tau_{l}$ along the refinement edge and mark the new triangles as indicated in Fig. 3. If a new node $V$ belongs to the discrete boundary, proceed as indicated in Fig. 4, i.e. set $V=\frac{V}{|V|}$. 
3. $l=l+1$, denote the new set of triangles with $\pi$

4. If $l=2 k$ then stop

5. Goto 2.

S2:

1. (a) $\tau_{0}:=\cup_{j=1}^{N+3} \Delta\left(0, e^{i \kappa_{j-1}}, e^{i \kappa_{j}}\right)$ (macro-triangulation, Fig. 1)

(b) $l=0$

2. Divide each $\Delta \in \pi$ into 4 congruent triangles as indicated in Fig 2. If a new node $V$ belongs to the discrete boundary, proceed as indicated in Fig. 4, i.e. set $V=\frac{V}{|V|}$.

3. $l=l+1$, denote the new set of triangles with $\tau_{l}$

4. If $l=k$ then stop

5. Goto 2 .

Clearly, both refinement strategies yield regular and conforming triangulations of $B$. We set

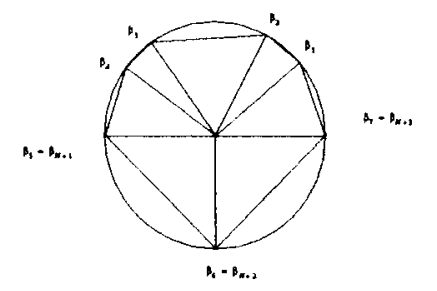

Figure 1: Macro-triangulation

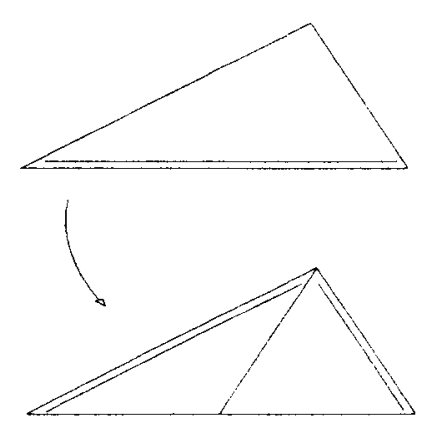

Figure 3: Bisection, refinement edges

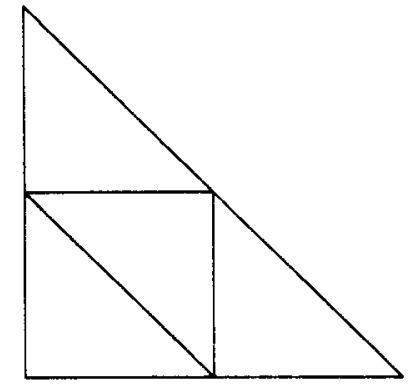

Figure 2: Congruent refinement

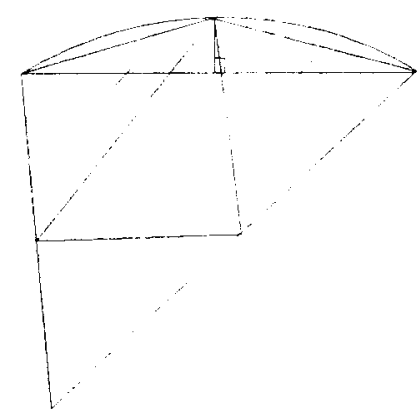

Figure 4: Boundary refinement.

$$
\begin{aligned}
B_{h} & :=\cup\{\Delta \in \tau\}, \\
Y_{h} & :=\left\{v \in C^{0}\left(\bar{B}_{h}, \mathbb{R}^{q}\right) ; v_{\left.\right|_{\Delta}} \text { linear } \forall \Delta \in \tau\right\},
\end{aligned}
$$


and consider $Y_{h}$ as a subspace of $H^{1}\left(B_{h}, \mathbb{R}^{q}\right)$. The discrete analogues of $X$ and $X_{0}$ are

$$
X_{h}:=\left\{v \in Y_{h} ; v\left(\bar{\gamma}_{k} \cap \partial B_{h}\right) \subset a_{k}+\Gamma_{k}(1 \leq k \leq N+3)\right\}
$$

and

$$
X_{h 0}:=\left\{v \in Y_{h} ; v\left(\bar{\gamma}_{k} \cap \partial B_{h}\right) \subset \Gamma_{k}(1 \leq k \leq N+3)\right\},
$$

respectively. For functions in $Y_{h}$ we define the discrete Dirichlet integral by

$$
D_{h}(v):=\frac{1}{2} \int_{B_{h}}|\nabla v|^{2} d x .
$$

In order to obtain the inclusion $X_{h} \subseteq X$ we have to define a suitable prolongation operator for functions in $Y_{h}$. Let $\eta$ denote the outer unit normal vector to $\partial B_{h}$. For $x \in \mathbb{R}^{2}$, let $a(x):=\operatorname{dist}\left(x, \partial B_{h}\right)$ measure the distance between $x$ and $\partial B_{h}$. The prolongation operator is defined as follows

$$
\begin{aligned}
& \text { Pro: } \quad Y_{h} \rightarrow H^{1}\left(B, \mathbb{R}^{q}\right) \\
& \operatorname{Pro}(v)(x):=\left\{\begin{aligned}
v(x), & x \in \bar{B}_{h} \\
v(x-a(x) \eta), & x \in \bar{B} \backslash \bar{B}_{h}
\end{aligned}\right.
\end{aligned}
$$

The definition of Pro implies that $\operatorname{Pro}(v) \in X$, if $v \in X_{h}$. Moreover, Pro is a continuous operator with respect to the $H^{1}$-topology (see [10, Bemerkung 3.8]). For what follows we introduce the convention of not distinguishing between $v$ and $\operatorname{Pro}(v)$.

The discrete analogue to the variational problem (16) is

$$
\Theta_{h}(\kappa)=\inf _{v \in X_{h}(\kappa)} D_{h}(v)
$$

This problem has a unique solution $u_{h}=u_{h}(\kappa) \in X_{h}$, called discrete quasiminimal surface that satisfies (see [11, Lemma 4.1.1])

$$
\int_{B_{h}} \nabla u_{h} \nabla v d x=0 \quad \forall v \in X_{h 0} .
$$

Next we prove a $H^{1}$-error estimate for discrete quasiminimal surfaces. For this purpose let $m \in \mathbb{N}$, $2<p \leq \infty, m-\frac{2}{p}>0$, let $h>0$ denote the gridsize of $\tau$ and set

$$
\begin{aligned}
I_{h}: H^{m, p}\left(B, \mathbb{R}^{q}\right) & \rightarrow Y_{h} \\
I_{h} v(x) & :=v(x) \text { at the nodes } x \text { of } \tau .
\end{aligned}
$$

The definition of $I_{h}$ clearly implies

$$
I_{h} v \in X_{h}\left(X_{h 0}\right) \text { if } v \in X\left(X_{0}\right) \cap C^{0}\left(\bar{B}, \mathbb{R}^{q}\right)
$$

Now let $u=u(\kappa)$ denote the solution of (16). The $I_{h}$-interpolant of $u$ satisfies (ser [11, Theorem 5.3])

$$
\left\|u-I_{h} u\right\|_{1, B_{h}} \leq c h^{\frac{p-2}{p}} \quad \forall 2 \leq p<-\frac{2}{p_{\min }}
$$

with some positive constant $c=c(u, \tau, N)$ and $p_{\min }$ from (26). This is the main tool in proving

Theorem 3.1 Let $h>0$ denote the gridsize of $\tau$ of and let $u . u_{h}$ be the unique solutions of (16) and (41), respectively. Then we have

$$
\left\|u-u_{h}\right\|_{1, B_{h}} \leq c h^{\frac{p-2}{\mathbf{p}}} \quad \forall 2 \leq p<-\frac{2}{p_{\text {min }}}
$$

where $c=c(u, \tau, N)>0$. 
Proof : With the help of Lemma 2.2, the continuity of the operator Pro and Eq. (45) we obtain (compare $[10$, p.38])

$$
\begin{aligned}
\left\|u-u_{h}\right\|_{B_{h}} & \leq\left\|u-I_{h} u\right\|_{B_{h}}+\left\|I_{h} u-u_{h}\right\|_{B_{h}} \\
& \leq c\left(h^{\frac{p-2}{p}}+\left|u-u_{h}\right|_{1, B_{h}}\right) .
\end{aligned}
$$

Obviously it remains to estimate $\left|u-u_{h}\right|_{1, B_{h}}$. The variational equalities (17) and (42) imply for $v \in X_{h}$

$$
\left|u-u_{h}\right|_{1, B_{h}}^{2}=\int_{B_{h}} \nabla\left(u-u_{h}\right) \nabla(u-v) d x-\int_{B \backslash B_{h}} \nabla u \nabla\left(v-u_{h}\right) d x .
$$

Using Young's inequality, a straightforward estimate yields

$$
\left|u-u_{h}\right|_{1, B_{h}} \leq c \inf _{v \in X_{h}}\left(|u-v|_{1, B_{h}}+|u|_{1, B \backslash B_{h}}\right) .
$$

Since meas $\left(B \backslash B_{h}\right) \leq c h^{2}$, it follows from Hölder's inequality that.

$$
\begin{aligned}
|u|_{1, B \backslash B_{h}} \leq \operatorname{meas}\left(B \backslash B_{h}\right)^{\frac{p-2}{2 p}}|u|_{1, p, B \backslash B_{h}} \\
\leq c h^{\frac{p-2}{p}}|u|_{1, p, B} \quad \forall 2 \leq p<-\frac{2}{p_{\min }} .
\end{aligned}
$$

Setting $v:=I_{h} u$ in (47), the assertion follows from (45).

For the convergence of Dirichlet's integral we can say more.

Corollary 3.1 With the notation of Theorem 3.1 the difference $D(u)-D\left(u_{h}\right)$ satisfies the estimate

$$
\left|D(u)-D_{h}\left(u_{h}\right)\right| \leq c h^{2 \frac{p-2}{p}} \quad \forall 2 \leq p<\frac{-2}{p_{m i n}}
$$

with some $c=c(u, \tau, N)>0$.

Proof : We write

$$
D(u)-D_{h}\left(u_{h}\right)=\underbrace{-\frac{1}{2} \int_{B_{h}}\left|\nabla\left(u-u_{h}\right)\right|^{2} d x}_{(1)}+\underbrace{\int_{B_{h}} \nabla u \nabla\left(u-u_{h}\right) d x}_{(2)}+\underbrace{\frac{1}{2} \int_{B \backslash B_{h}}|\nabla u|^{2} d x}_{(3)}
$$

and set $\mu:=\frac{p-2}{p}$. Equation 45 together with meas $\left(B \backslash B_{h}\right)=O\left(h^{2}\right)$ yields

$$
|(1),(3)| \leq c h^{2 \mu} \quad \forall 2 \leq p<\frac{-2}{p_{\min }} .
$$

In order to estimate (2) we recall that the operator Pro defined in (40) is continuous, satisfies Pro $\left(u_{h}\right) \in X$ and $\nabla u_{h_{1 T}} \equiv$ const on every triangle $T$ of the triangulation $\tau_{h}$. The variational equality (17) therefore implies

$$
\begin{aligned}
\int_{B_{h}} \nabla u \nabla\left(u-u_{h}\right) d x & =-\int_{B \backslash B_{h}} \nabla u \nabla\left(u-u_{h}\right) d x \\
& =-\int_{B \backslash B_{h}}|\nabla u|^{2} d x+\int_{B \backslash B_{h}} \nabla u \nabla u_{h} d x .
\end{aligned}
$$

Thus

$$
|(2)| \leq c h^{2 \mu}+\int_{B \backslash B_{h}}\left|\nabla u \nabla u_{h}\right| d x
$$


and, using the Cauchy-Schwarz inequality

$$
\begin{aligned}
\int_{B \backslash B_{h}}\left|\nabla u \nabla u_{h}\right| d x & \leq\left(\int_{B \backslash B_{h}}|\nabla u|^{2} d x\right)^{\frac{1}{2}}\left(\int_{B \backslash B_{h}}\left|\nabla u_{h}\right|^{2} d x\right)^{\frac{1}{2}} \\
& \leq c h^{\mu}\left(\int_{B \backslash B_{h}}\left|\nabla u_{h}\right|^{2} d x\right)^{\frac{1}{2}} .
\end{aligned}
$$

Now it remains to estimate $\int_{B \backslash B_{h}}\left|\nabla u_{h}\right|^{2} d x$. For this purpose define $\Omega^{*}$ to be the union of these triangles of the triangulation $\tau_{h}$ having a boundary edge and denote for a triangle $T \subset \Omega^{*}$ by $\Omega_{T}$ the segment defined by $T$ and $\partial B$. Then we have

$$
\begin{aligned}
\int_{B \backslash B_{h}}\left|\nabla u_{h}\right|^{2} d x & =\sum_{T \subset \Omega^{*}} \int_{\Omega_{T}}\left|\nabla u_{h}\right|^{2} d x \leq \sum_{T \subset \Omega^{*}} \underbrace{\operatorname{meas}\left(\Omega_{T}\right)}_{=O\left(h^{3}\right)}\left|\nabla\left(u_{h_{1 T}}\right)\right|^{2} \\
& \leq c h \sum_{T \subset \Omega^{*}} \int_{T}\left|\nabla u_{h}\right|^{2} d x=c h \int_{\Omega^{\cdot}}\left|\nabla u_{h}\right|^{2} d x .
\end{aligned}
$$

Since meas $\left(\Omega^{*}\right) \leq c(N) h$ Hölder's inequality yields

$$
\begin{aligned}
\int_{\Omega^{*}}\left|\nabla u_{h}\right|^{2} d x & =\int_{\Omega^{*}}|\nabla u|^{2} d x+\int_{\Omega^{*}}\left|\nabla\left(u-u_{h}\right)\right|^{2} d x-2 \int_{\Omega^{*}} \nabla u \nabla\left(u-u_{h}\right) d x \\
& \leq c h^{\mu}+c h^{2 \mu}+2\left(\int_{\Omega^{*}}|\nabla u|^{2} d x\right)^{\frac{1}{2}}\left(\int_{\Omega^{*}}\left|\nabla\left(u-u_{h}\right)\right|^{2} d x\right)^{\frac{1}{2}} \\
& \leq c h^{\mu} \quad \forall 2 \leq p<\frac{-2}{p_{\min }} .
\end{aligned}
$$

We conclude

$$
\begin{array}{ll}
\left(\int_{B \backslash B_{h}}\left|\nabla u_{h}\right|^{2} d x\right)^{\frac{1}{2}} \leq c h^{\frac{1+\mu}{2}} \quad \forall 2 \leq p<\frac{-2}{p_{\min }}, \\
\int_{B \backslash B_{h}}\left|\nabla u \nabla u_{h}\right| d x \leq c h^{\frac{1+3 \mu}{2}} \quad \forall 2 \leq p<\frac{-2}{p_{\min }}
\end{array}
$$

and finally, since $h^{\frac{1+3 \mu}{2}} \leq h^{2 \mu} \forall 0<h<1$

$$
|(2)| \leq c h^{2 \frac{p-2}{p}} \quad \forall 2 \leq p<\frac{-2}{p_{\min }} .
$$

This completes the proof.

Remark 3.1 In the case $q \leq 3$ we have $p_{\min }=\min _{1 \leq k \leq N+3}\left\{-\alpha_{k}, \alpha_{k}-1\right\}$. when $\alpha_{k} \pi$ defined by (25)) denotes the outer angle at the vertex $a_{k}$ of the polygon $\Gamma(1 \leq k \leq N+3)$. In this case we have a direct relation between the smallest angle of the polygon $\Gamma$ and the order of convergenct.

By our choice of triangulations $\tau(\kappa)$ of the unit disc (the points $\beta_{j}(\kappa)=\epsilon^{i \kappa j}$ are nodes of the macrotriangulation) and Corollary 2.1 the constants in (45). (46) and (48) can be controlled uniformly for $\kappa \in T^{\prime} \subset \subset T$ ([11, Section 4.5]). This proves the following theorem that will be the main tool in proving existence and convergence of discrete minimal surfaces in the next section.

Theorem 3.2 Let $T^{\prime} \subset \subset T$ and let $h>0$ denote the gridsize of $\tau_{h}(\kappa)$. Let $u_{h}(\kappa), u(\kappa)^{\prime}$ denote the solutions of (41) and (16), respectively. Then we have

$$
\sup _{\kappa \in T^{\prime}}\left\|u(\kappa)-u_{h}(\kappa)\right\|_{1, B_{h}} \leq c_{1} h^{\frac{p-2}{p}} \quad \forall 2 \leq p<-\frac{2}{p_{\min }}
$$

and

$$
\left\|\Theta-\Theta_{h}\right\|_{\infty, T^{\prime}} \leq c_{2} h^{2 \frac{p-2}{p}} \quad \forall 2 \leq p<\frac{-2}{p_{\min }}
$$

with constants $c_{i}=c_{i}\left(T^{\prime}\right)>0(i=1,2)$. 


\section{Existence and convergence of discrete minimal surfaces}

We start with defining an approximation of $\nabla \Theta$, which was defined in (33). For this purpose we set

$$
a(x):=\frac{x_{1}^{2}-x_{2}^{2}}{x_{1}^{2}+x_{2}^{2}}, \quad b(x):=\frac{x_{1} x_{2}}{x_{1}^{2}+x_{2}^{2}}, \quad x \in \bar{B} \backslash\{0\}
$$

and note that $a$ and $b$ are uniformly bounded on $\bar{B}$. Next we set $S_{h}\left(\kappa_{k}, \kappa_{k+1}\right):=S\left(\kappa_{k}, \kappa_{k+1}\right) \cap B_{h}$ (see (33) and define a function $f_{h}: T \rightarrow \mathbb{R}^{N}, f_{h}=\left(f_{h}^{1}, \ldots, f_{h}^{N}\right)^{t}$ in terms of their components by

$$
\begin{aligned}
& f_{h}^{k}(\kappa):=-\frac{1}{2}\left(\frac{1}{\kappa_{k+1}-\kappa_{k}} \int_{S_{h}\left(\kappa_{k}, \kappa_{k+1}\right)}\left(u_{h x_{1}}^{2}(\kappa)-u_{h x_{2}}^{2}(\kappa)\right) a(x)+4 u_{h x_{1}}(\kappa) u_{h x_{2}}(\kappa) b(x) d x_{1} d x_{2}-\right. \\
& \left.\frac{1}{\kappa_{k}-\kappa_{k-1}} \int_{S_{h}\left(\kappa_{k-1}, \kappa_{k}\right)}\left(u_{h x_{1}}^{2}(\kappa)-u_{h_{x_{2}}}^{2}(\kappa)\right) a(x)+4 u_{h_{s_{1}}}(\kappa) u_{h s_{2}}(\kappa) b(x) d x_{1} d x_{2}\right)(k=1, \ldots, N) .
\end{aligned}
$$

In this expression $u_{h}(\kappa)$ denotes the discrete quasiminimal surfaces corresponding to a $\kappa \in T$.

The next theorem proves that $f_{h}$ is in fact an approximation of $\nabla \Theta$.

Theorem 4.1 Let $f_{h}(\kappa)$ be defined by (51). Then we have for every $T^{\prime} \subset \subset T$

$$
\left\|\nabla \Theta-f_{h}\right\|_{\infty, T^{\prime}} \leq \operatorname{ch}^{\frac{p-2}{p}} \quad \forall 2 \leq p<\frac{-2}{p_{\min }}
$$

with a positive constant $c=c\left(T^{\prime}\right)$ and $p_{\min }>-1$ from (26).

Proof : Taking the difference of (33) and (51), it remains to estimate integrals of the form

$$
\int_{S_{h}}\left(u_{h_{x_{i}}}^{2}(\kappa)-u_{x_{i}}^{2}(\kappa)\right) h(x) d x \quad, \int_{S \backslash S_{h}} u_{x_{i}}^{2}(\kappa) h(x) d x \quad(i=1.2),
$$

and respectively. But

$$
\left.\int_{S_{h}}\left(u_{h_{x_{1}}}(\kappa) u_{h_{x_{2}}}(\kappa)-u_{x_{1}}(\kappa) u_{x_{2}}(\kappa)\right) g(x) d x, \quad \int_{S \backslash S_{h}} u_{x_{1}}(\kappa) u_{x_{2}}(\kappa)\right) g(x) d x
$$

$$
\begin{gathered}
u_{h_{x_{i}}}^{2}(\kappa)-u_{x_{i}}^{2}(\kappa)=\left(u_{h_{x_{i}}}(\kappa)-u_{x_{i}}(\kappa)\right)^{2}+2 u_{x_{1}}\left(u_{h_{x_{i}}}(\kappa)-u_{x_{1}}(\kappa)\right) \quad(i=1,2), \\
u_{h_{x_{1}}}(\kappa) u_{h_{x_{2}}}(\kappa)-u_{x_{1}}(\kappa) u_{x_{2}}(\kappa)=\left(u_{h_{x_{1}}}(\kappa)-u_{x_{1}}(\kappa)\right) u_{x_{2}}+\left(u_{h_{x_{2}}}(\kappa)-u_{x_{2}}(\kappa)\right) u_{x_{1}} .
\end{gathered}
$$

and meas $\left(S \backslash S_{h}\right)=O\left(h^{2}\right)$. Thus estimate (49) gives the desired result.

According to Theorem 4.1 it makes sense to define discrete minimal surfaces spanning a polygon $\Gamma \subset \mathbb{R}^{q}$ in the following way

Definition 4.1 Let $\kappa_{h}^{*} \in T$ satisfy $f_{h}\left(\kappa_{h}^{*}\right)=0$. The corresponding quasiminimal surface $u_{h}\left(k_{h}^{*}\right)$ is called discrete minimal surface.

In what follows we prove the existence of discrete minimal surfaces in neighbourhoods of minimal surfaces corresponding to regular critical points of the function $\Theta$. For this purpose let $\kappa^{*} \in T$ with $\operatorname{rank} D^{2} \Theta\left(\kappa^{*}\right)=N$ and set

$$
s_{h}(\kappa):=\kappa-D^{2} \Theta\left(\kappa^{*}\right)^{-1} f_{h}(\kappa), \quad \kappa \in T .
$$

Then clearly every fixed point of $s_{h}$ in $T$ is a root of the vector function $f_{h}$.

By Lemma 2.1 a neighbourhood $V_{e_{0}}\left(\kappa^{*}\right) \subset T$ exists, such that

$$
\nabla \Theta(\kappa) \neq 0 \quad \forall \kappa \in V_{\varepsilon 0}\left(\kappa^{*}\right) \backslash\left\{\kappa^{*}\right\} .
$$

In order to apply the Brouwer fixed point theorem to the function $s_{h}$ we prove 
Lemma 4.1 There exists $a$ constant $\tilde{c}>0$ and $a h_{0}>0$ such that for all $0<h \leq h_{0}$

$$
s_{h}\left(\overline{V_{Q h}\left(\kappa^{*}\right)}\right) \subset V_{Q_{h}}\left(\kappa^{*}\right) \subset V_{Q_{0}}\left(\kappa^{*}\right)
$$

holds, where $\varrho_{h}:=\tilde{c} h^{\frac{p-2}{p}}, 2<p<\frac{-2}{p_{m i n}}, p_{\min }$ from (26)

Proof : The definition of $s_{h}$ implies

$$
s_{h}(\kappa)-\kappa^{*}=D^{2} \Theta\left(\kappa^{*}\right)^{-1}\left\{D^{2} \Theta\left(\kappa^{*}\right)\left(\kappa-\kappa^{*}\right)-\nabla \Theta(\kappa)\right\}+D^{2} \Theta\left(\kappa^{*}\right)^{-1}\left\{\nabla \Theta(\kappa)-f_{h}(\kappa)\right\} .
$$

Estimate (52) applied to the difference $D^{2} \Theta\left(\kappa^{*}\right)^{-1}\left\{\nabla \Theta(\kappa)-f_{h}(\kappa)\right\}$ yields the existence of a positive constant $c=c\left(\overline{V_{\varrho_{0}}\left(\kappa^{*}\right)}\right)$ such that

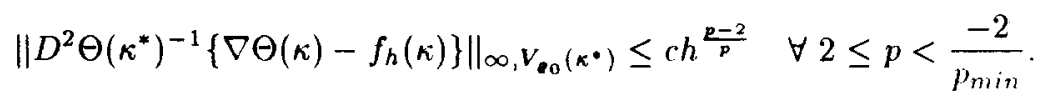

Now we define $\tilde{c}:=2 c$ and note that since $\Theta$ is analytic in $\mathrm{T}$ and $\nabla \Theta\left(\kappa^{*}\right)=0$

$$
D^{2} \Theta\left(\kappa^{*}\right)\left(\kappa-\kappa^{*}\right)-\nabla \Theta(\kappa)=o\left(\left|\kappa-\kappa^{*}\right|\right)\left(\kappa \rightarrow \kappa^{*}\right)
$$

holds. Therefore we can choose $0<h_{0}<\varrho_{0}$ in such a way that for all $0<h \leq h_{0}, 2 \leq p<\frac{-2}{p_{m ! n}}$

$$
\left|\kappa-\kappa^{*}\right| \leq \tilde{c} h^{\frac{p-2}{p}} \Rightarrow\left|D^{2} \Theta\left(\kappa^{*}\right)^{-1}\left\{D^{2} \Theta\left(\kappa^{*}\right)\left(\kappa-\kappa^{*}\right)-\nabla \Theta(\kappa)\right\}\right|<\frac{\bar{c}}{2} h^{\frac{p-z}{p}} .
$$

Now set $\varrho_{h}:=\tilde{c} h^{\frac{p-2}{p}}$ and let $\kappa \in V_{\varrho_{h}}\left(\kappa^{*}\right)$. Then Eqs. (5.3) and (54) imply

$$
\left|s_{h}(\kappa)-\kappa^{*}\right|<\left(\frac{\tilde{c}}{2}+c\right) h^{\frac{p-2}{p}}=c_{h}^{\frac{p-2}{p}} \quad \forall 2 \leq p<\frac{-2}{p_{\min }},
$$

i.e. $s_{h}\left(\overline{V_{\ell h}\left(\kappa^{*}\right)}\right) \subset V_{\ell_{h}}\left(\kappa^{*}\right) \subset V_{\ell 0}\left(\kappa^{*}\right)$. This proves the lemma.

Since the function $f_{h}(\kappa)$ is continuous and $\overline{V_{Q_{h}}\left(\kappa^{*}\right)}$ is compact and convex, we are in the position to apply Brouwer's fixed point theorem to $s_{h}$. We conclude that there exists at least one fixed point $\kappa_{h}^{*}$ of $s_{h}$ in $V_{\varrho_{h}}\left(\kappa^{*}\right)$, i.e. $f_{h}\left(\kappa_{h}^{*}\right)=0$. For $\kappa=\kappa_{h}^{*}$ Eq. (55) now yields

$$
\left|s_{h}\left(\kappa_{h}^{*}\right)-\kappa^{*}\right|=\left|\kappa_{h}^{*}-\kappa^{*}\right| \leq \tilde{c} h^{\frac{\mathrm{p}-2}{\mathrm{p}}} \quad \forall 2 \leq p<\frac{-2}{p_{\min }} .
$$

Thus, we have proved

Theorem 4.2 Let $\kappa^{*} \in T$ be a critical point of $\Theta$ such that rank $D^{2} \Theta\left(\kappa^{*}\right)=N$. Then there exists a neighbourhood $V\left(\kappa^{*}\right) \subset T$ and $a h_{0}>0$ so that for all $0<h \leq h_{0}$ the equation

$$
f_{h}(\kappa)=0
$$

has at least one solution $\kappa_{h}^{*} \in V\left(\kappa^{*}\right)$ which satisfies (56).

This theorem now implies the convergence of the sequence $\left\{\kappa_{h}^{*}\right\}_{0<h \leq h_{0}}$ to $\kappa^{* *}$. In the following theorem we prove convergence of the discrete minimal surfaces $u_{h}\left(\kappa_{h}^{*}\right)$ to $u\left(\kappa^{*}\right)(h \rightarrow 0)$ in the $H^{1}$-norm and convergence of the corresponding Dirichlet integrals.

Theorem 4.3 Let $\kappa^{*} \in T$ be a regular critical point of $\Theta$ and let $u\left(\kappa^{*}\right)$ denote the corresponding minimal surface. Furthermore, let $\left\{\kappa_{h}^{*}\right\}_{0<h \leq h_{0}}$ denote the sequence from Theorem 4. and let $u_{h}\left(\kappa_{h}^{*}\right)$ denote the corresponding discrete minimal surfaces. Then

$$
\left\|u\left(\kappa^{*}\right)-u_{h}\left(\kappa_{h}^{*}\right)\right\|_{1, B_{h}} \rightarrow 0 \quad(h \rightarrow 0)
$$

and

$$
\left|D\left(u\left(\kappa^{*}\right)\right)-D_{h}\left(u_{h}\left(\kappa_{h}^{*}\right)\right)\right| \leq c h^{2 \frac{p-2}{p}} \quad \forall 2 \leq p<\frac{-2}{p_{\min }}
$$

holds. The constant $c$ is positive and depends on $V\left(\kappa^{*}\right)$ from Theort 4.2 . 
Proof : We write

$$
\left\|u\left(\kappa^{*}\right)-u_{h}\left(\kappa_{h}^{*}\right)\right\|_{1, B_{h}} \leq \underbrace{\left\|u\left(\kappa^{*}\right)-u\left(\kappa_{h}^{*}\right)\right\|_{1, B}}_{(1)}+\underbrace{\left\|u\left(\kappa_{h}^{*}\right)-u_{h}\left(\kappa_{h}^{*}\right)\right\|_{1, B_{h}}}_{(2)}
$$

Since $\left\{\kappa_{h}^{*}\right\}_{0<h \leq h_{0}} \subset V\left(\kappa^{*}\right) \subset \subset T$ the second term can be estimated using (49),

$$
(2) \leq \operatorname{ch}^{\frac{p-2}{p}} \quad \forall 2 \leq p<\frac{-2}{p_{\min }}
$$

$\left\{u\left(\kappa_{h}^{*}\right)\right\}_{0<h \leq h_{0}} \subset H^{1}\left(B, \mathbb{R}^{q}\right)$ is uniformly bounded by $(30)$. Therefore, at least for subsequences, which will also be denoted by $\left\{u\left(\kappa_{h}^{*}\right)\right\}_{0<h \leq h_{0}}$ we have

$$
\begin{aligned}
& u\left(\kappa_{h}^{*}\right) \rightarrow v \quad \text { in } H^{1}\left(B, \mathbb{R}^{q}\right) \\
& u\left(\kappa_{h}^{*}\right) \rightarrow v \quad \text { in } L^{2}\left(B, \mathbb{R}^{q}\right)
\end{aligned}
$$

and

$$
u\left(\kappa_{h}^{*}\right) \rightarrow v \text { in } L^{2}\left(\partial B, \mathbb{R}^{q}\right) \quad\left(\Rightarrow v \in X\left(\kappa^{*}\right)\right)
$$

Since

$$
D\left(u\left(\kappa^{*}\right)\right)=\Theta\left(\kappa^{*}\right)=\lim _{h \rightarrow 0} \Theta\left(\kappa_{h}^{*}\right)=\lim _{h \rightarrow 0} D\left(u\left(\kappa_{h}^{*}\right)\right) \geq \liminf _{h \rightarrow 0} D\left(u\left(\kappa_{h}^{*}\right)\right) \geq D(v)
$$

and $u\left(\kappa^{*}\right)$ is uniquely determined by $\kappa^{*}$, we conclude that $v=u\left(\kappa^{*}\right)$. A well known theorem ([1, Übung 5.5]) now implies

$$
u\left(\kappa_{h}^{*}\right) \rightarrow u\left(\kappa^{*}\right) \text { in } H^{1}\left(B, \mathbb{R}^{q}\right) \text {, i.e. (1) } \rightarrow 0 \text {. }
$$

Moreover, the whole sequence $\left\{u\left(\kappa_{h}^{*}\right)\right\}_{0<h \leq h_{0}}$ converges to $u\left(\kappa^{*}\right)$, for otherwise we could select a subsequence converging to another limit. Combining (59) and (60) we finally obtain

$$
\left\|u\left(\kappa^{*}\right)-u_{h}\left(\kappa_{h}^{*}\right)\right\|_{1, B_{h}} \rightarrow 0 \quad(h \rightarrow 0)
$$

With respect to the convergence of the Dirichlet integrals $D\left(u_{h}\left(\kappa_{h}^{*}\right)\right)$ we can show more. We write

$$
\left|D\left(u\left(\kappa^{*}\right)\right)-D_{h}\left(u_{h}\left(\kappa_{h}^{*}\right)\right)\right| \leq \underbrace{\left|D\left(u\left(\kappa^{*}\right)\right)-D\left(u\left(\kappa_{h}^{*}\right)\right)\right|}_{(1)}+\underbrace{\left|D_{h}\left(u\left(\kappa_{h}^{*}\right)\right)-D_{h}\left(u_{h}\left(\kappa_{h}^{*}\right)\right)\right|}_{(2)}+\underbrace{\int_{B \backslash B_{h}}\left|\nabla u\left(\kappa_{h}^{*}\right)\right|^{2} d x}_{(3)} .
$$

Since $\Theta$ is analytic in $T$ and $\nabla \Theta\left(\kappa^{*}\right)=0$ Taylor's theorem implies

$$
\begin{aligned}
(1) & \leq \frac{1}{2}\left|D^{2} \Theta\left(\kappa^{*}\right)\right|\left|\kappa_{h}^{*}-\kappa^{*}\right|^{2}+o\left(\left|\kappa_{h}^{*}-\kappa^{*}\right|^{2}\right)(h \rightarrow 0) \\
& \leq c h^{2 \frac{p-2}{p}} \quad \forall 2 \leq p<\frac{-2}{p_{\min }}
\end{aligned}
$$

with some positive constant $c=c\left(\overline{V\left(\kappa^{*}\right)}\right)$.

The second term can be estimated using (50),

$$
(2) \leq c h^{2 \frac{p-2}{p}} \quad \forall 2 \leq p<\frac{-2}{p_{\min }} .
$$

Applying Hölder's inequality, (30) finally yields

$$
(3) \leq c \underbrace{\operatorname{meas}\left(B \backslash B_{h}\right)^{\frac{p-2}{p}}}_{\leq c h^{2}} \underbrace{\left|u\left(\kappa_{h}^{*}\right)\right|_{1, p, B}}_{\leq c}
$$


with some positive constant $c=c\left(\overline{V\left(\kappa^{*}\right)}\right)$. Hence,

$$
\left|D\left(u\left(\kappa^{*}\right)\right)-D_{h}\left(u_{h}\left(\kappa_{h}^{*}\right)\right)\right| \leq c h^{2 \frac{p-2}{p}} \quad \forall 2 \leq p<\frac{-2}{p_{\text {min }}} .
$$

This completes the proof.

The following corollary is a refinement of (57).

Corollary 4.1 Under the assumptions of Theorem 4.3

$$
\left\|u\left(\kappa^{*}\right)-u_{h}\left(\kappa_{h}^{*}\right)\right\|_{1, B_{h}} \leq c h^{\left(\frac{p}{p-2}\right)^{2}} \quad \forall 2 \leq \mu<\frac{-2}{p_{\min }}
$$

holds. The constant $c=c\left(V\left(\kappa^{*}\right)\right)$ is positive.

Proof : Since $\beta_{k}\left(\kappa_{N+i}^{*}\right)=\beta_{k}\left(\kappa_{h_{N+}}^{*}\right)(i=1,2,3)$ Lemma 2.2 gives

$$
\left\|u\left(\kappa^{*}\right)-u_{h}\left(\kappa_{h}^{*}\right)\right\|_{1, B_{h}} \leq c \underbrace{\left|u\left(\kappa^{*}\right)-u\left(\kappa_{h}^{*}\right)\right|_{1, B}}_{(1)}+\underbrace{\left\|u\left(\kappa_{h}^{*}\right)-u_{h}\left(\kappa_{h}^{*}\right)\right\|_{1, B_{h}}}_{(2)} .
$$

For (2) estimate (59) holds. In order to estimate (1) we write

$$
\frac{1}{2}|(1)|^{2} \leq \underbrace{\left|D\left(u\left(\kappa_{h}^{*}\right)\right)-D\left(u\left(\kappa^{*}\right)\right)\right|}_{(3)}+\mid \underbrace{\int_{B} \nabla u\left(\kappa^{*}\right) \nabla\left(u\left(\kappa^{*}\right)-u\left(\kappa_{h}^{*}\right)\right) d x \mid}_{(4)} .
$$

As in the proof of the previous theorem we conclude

$$
|(3)| \leq c h^{2 \frac{p}{p-2}} \quad \forall 2 \leq p<\frac{-2}{p_{\min }} .
$$

Integration by parts applied to (4) together with (18) yields

$$
\begin{gathered}
(4)=\int_{\partial B} u_{r}\left(\kappa^{*}\right)\left(u\left(\kappa^{*}\right)-u\left(\kappa_{h}^{*}\right)\right) d s= \\
\sum_{k=1}^{N+3}(\underbrace{\int_{\gamma_{k}\left(\kappa^{*}\right) \cap \gamma_{k}\left(\kappa_{h}^{*}\right)} u_{r}\left(\kappa^{*}\right)\left(u\left(\kappa^{*}\right)-u\left(\kappa_{h}^{*}\right)\right) d s}_{(5) \gamma_{k}}+\underbrace{\left.\int_{\left(\gamma_{k}\left(\kappa^{*}\right) \backslash \gamma_{k}\left(\kappa_{h}^{*}\right)\right) \cup\left(\gamma_{k}\left(\kappa_{h}^{*}\right) \backslash \gamma_{k}\left(\kappa^{*}\right)\right)} u_{r}\left(\kappa^{*}\right)\left(u\left(\kappa^{*}\right)-u\left(\kappa_{h}^{*}\right)\right) d s\right)}_{(f)_{k}} .
\end{gathered}
$$

Since

$$
u\left(\kappa^{*}\right)-u\left(\kappa_{h}^{*}\right) \in a_{k}+\Gamma_{k}, w \in \gamma_{k}\left(\kappa^{*}\right) \cap \gamma_{k}\left(\kappa_{h}^{*}\right),
$$

Eq. (20) gives $(5)_{k}=0(k=1, \ldots, N+3)$. To estimate $(6)_{k}$ we note that by $(56)$

$$
\operatorname{meas}\left(\left(\gamma_{k}\left(\kappa^{*}\right) \backslash \gamma_{k}\left(\kappa_{h}^{*}\right)\right) \cup\left(\gamma_{k}\left(\kappa_{h}^{*}\right) \backslash \gamma_{k}\left(\kappa^{*}\right)\right)\right) \leq c h^{\frac{p-2}{p}} \quad \forall 2 \leq p<\frac{-2}{p_{\min }} .
$$

Using expansion (23) we arrive at

$$
|(4)| \leq c(N) h^{\left(\frac{p-2}{p}\right)^{2}} \underbrace{\left\|u\left(\kappa^{*}\right)-u\left(\kappa_{h}^{*}\right)\right\|_{\sim, B}}_{(7)} .
$$

Combining (23), Taylor's theorem and (56) we finally obtain

$$
(7) \leq c h^{\left(\frac{p-2}{p}\right)^{2}} \quad \forall 2 \leq p<\frac{-2}{p_{\min }}
$$

and thus the desired result. 


\section{Examples of computed discrete minimal surfaces}

In the present chapter we present numerical investigations on generalized polygonal minimal surfaces Our starting point is the vector function $\nabla \Theta$. Due to Theorem 2.2 polygonal minimal surfaces $u\left(\kappa^{*}\right)$ (with Shiffman boundary conditions) are characterized by the equation $\nabla \Theta\left(\kappa^{*}\right)=0$. For this reason the computation of a polygonal minimal surface $u\left(\kappa^{*}\right)$ is equivalent to the computation of the corresponding critical value $\kappa^{*} \in T$ of $\Theta$. Moreover, all minimal surfaces spanning a polygon $\Gamma \subset \mathbb{R}^{q}$ can be obtained in this way.

According to Theorem 4.1 the function $f_{h}$ defined in (51) is well suited to substituting $\nabla \Theta$.

All computations in the present section were carried out by applying a damped Newton method to the equation

$$
f_{h}(\kappa)=0, \quad(\kappa \in T) .
$$

For an extensive discussion of the numerical implementation of $(62)$ we refer to [12, C'hapter 5$]$.

Example 5.1 The Riemann - Schwarz Surface, experimental order of convergenct (EOC)

The uniquely determined Plateau solution $u$ spanning the polygon $\Gamma \subset \mathbb{R}^{3}$ clefined by the vertices

$$
a_{1}\left(\frac{1}{2}, 0, \frac{1}{2 \sqrt{2}}\right), a_{2}\left(0,-\frac{1}{2},-\frac{1}{2 \sqrt{2}}\right), a_{3}\left(-\frac{1}{2}, 0, \frac{1}{2 \sqrt{2}}\right), a_{4}\left(0, \frac{1}{2},-\frac{1}{2 \sqrt{2}}\right) .
$$

is called Riemann - Schwarz Surface. This surface was already been investigated numerically by Jarausch in [14, Abb. 12, Abb. 27] and is therefore well suited for a comparismn of numerical results.

The area of the surface is $A(u)=0.63963 \pm 3 \cdot 10^{-5}$ and coincides with the value of Dirichlet's integral $D(u)$. At the corners of the polygon $u$ maps into the angle $\Varangle\left(\varphi_{\mathrm{k}},-\varphi_{k-1}\right)$, where $\varphi_{k}(k=1, \ldots N+3)$ was defined in (7). For the Dirichlet integrals of discrete minimal surfaces $u_{h}\left(\kappa_{h}^{*}\right)$ corresponding to parameter tuples $\kappa_{h}^{*} \in T$ satisfying $f_{h}\left(\kappa_{h}^{*}\right)=0$ we proved asymptotic convergence of order $O\left(h^{2 \mu-\epsilon}\right)(\epsilon>0)$, where

$$
\mu=\frac{1}{\pi} \Varangle\left(\varphi_{\mathrm{k}},-\varphi_{\mathrm{k}-1}\right)=\frac{1}{3}
$$

For the error

$$
E(h)=\left|D(u)-D_{h}\left(u_{h}\left(\kappa_{h}^{*}\right)\right)\right|
$$

the experimental order of convergence EOC between two gridsizes $h_{1} \neq h_{2}$ is given by

$$
E O C:=\frac{\ln \frac{E\left(h_{1}\right)}{E\left(h_{2}\right)}}{\ln \frac{h_{1}}{h_{2}}} \approx 2 \mu .
$$

As the following Table shows, the numerical results confirm the asymptotic convergence of Dirichlet's integral. The areas $A_{h}\left(u_{h}\right)$ of the discrete surfaces seem to converge faster than the values of the corresponding Dirichlet integrals.

Jarausch introduced polar coordinates on $B$ and approximated this surface using piecewise bilinear continuous finite elements. Jarausch's best approximation of the Riemann - Schwarz Surface has the area $A=0.640007$, the corresponding value of Dirichlet's integral is $D=0.675515$. This values nearly coincides with our values for $R L=5$.

In Figs. 6, 5 an approximation $u_{h}\left(\kappa_{h}^{*}\right)$ of the Riemann - Schwar surface together with corresponding triangulation are shown. 


\begin{tabular}{|r|r|r|r|c|c|c|c|}
\hline \multicolumn{10}{|c|}{ EOC for the Riemann - Schwarz Surface } \\
\hline$R L$ & $N V$ & $N T$ & $N R$ & $A_{h}\left(u_{h}\right)-A(u)$ & $E O C$ & $D_{h}\left(u_{h}\right)-D(u)$ & $E O C$ \\
\hline \hline 1 & 13 & 16 & 8 & $2.3280 \mathrm{e}-2$ & - & $2.0682 \mathrm{e}-1$ & - \\
\hline 2 & 41 & 64 & 16 & $7.9732 \mathrm{e}-3$ & 1.79 & $1.5917 \mathrm{e}-1$ & 0.44 \\
\hline 3 & 145 & 256 & 32 & $2.9080 \mathrm{e}-3$ & 1.58 & $1.0698 \mathrm{e}-1$ & 0.62 \\
\hline 4 & 545 & 1024 & 64 & $1.0978 \mathrm{e}-3$ & 1.46 & $6.8946 \mathrm{e}-2$ & 0.66 \\
\hline 5 & 2113 & 4096 & 128 & $4.2100 \mathrm{e}-4$ & 1.41 & $4.3741 \mathrm{e}-2$ & 0.67 \\
\hline 6 & 8321 & 16384 & 256 & $1.6290 \mathrm{e}-4$ & 1.38 & $2.7585 \mathrm{e}-2$ & 0.67 \\
\hline 7 & 33025 & 65536 & 512 & $6.3600 \mathrm{e}-5$ & 1.36 & $1.7360 \mathrm{e}-2$ & 0.67 \\
\hline
\end{tabular}

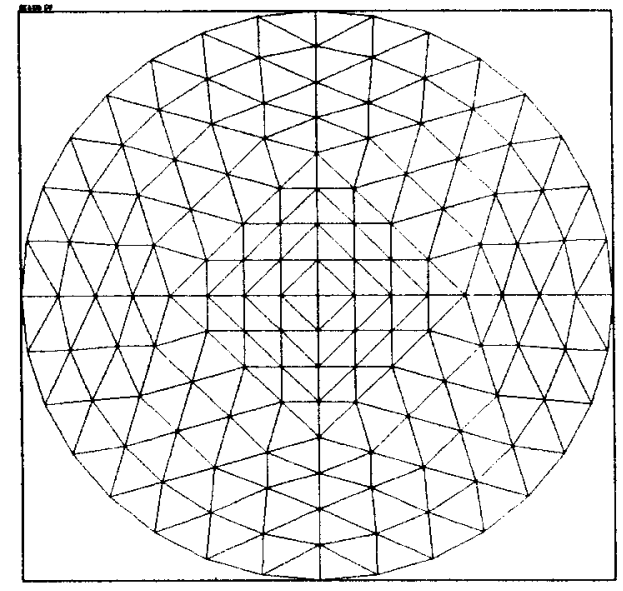

Figure 5: Triangulation

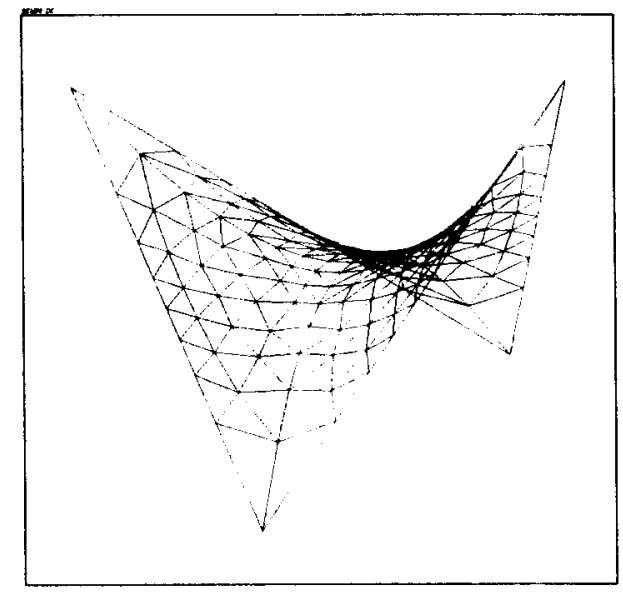

Figure 6: Riemann - Schwarz Surface

Example 5.2 Minimal surface spanning a polygon of Lewerenz

We consider the family of contours $\left\{P_{\epsilon}^{2}\right\}_{t>0}$ defined by the vertices

$$
a_{1}(0,0, \epsilon), a_{2}(1,1,-1), a_{3}(0,2,0), a_{4}(-1,1,1), a_{5}(0,0,-\epsilon)(\epsilon>0) .
$$

that was constructed by Lewerenz to prove that Courant's function $d$ and Shiffman's function $\Theta$ do not coincide for certain values of $\kappa$ (see [15, Satz]). Here we recall that quasiminimal surfaces $u(\kappa)$ are not required to map the closed arc $\bar{\gamma}_{k}$ onto the segment $\left[a_{k}, a_{k+1}\right]$ on the line $a_{k}+I_{k}(1 \leq k \leq N+3)$ in a monotone way, but instead may overshoot the vertices $a_{k}, a_{k+1}$.

In Figs. 7,8 we present the polygon $P_{2^{-6}}$ that bounds a discrete quasiminimal surface $u\left(\kappa_{h}\right)$ that overshoots the vertices of the polygon and is not order preserving on the boundary nodes of the corresponding triangulation of the unit disc. The parameter tuple $\kappa_{h}$ was used as the initial guess for the Newton process. In Figs. 9, 10 we present the discrete minimal surface $u_{h}\left(\kappa_{h}^{*}\right)$ spanning $P_{2-6}$ together with the triangulation $\tau_{h}\left(\kappa_{h}^{*}\right)$. The area of the discrete surface is $A=2.8136$, Dirichlet's integral has the value $D=2.9426$. The triangulation contains $N T=1280$ triangles, $N V=681$ nodes and $N R=80$ boundary nodes.

As Fig. 11 shows, the discrete minimal surface does not overshoot the vertices of the polygon and is order preserving on $\{$ boundary nodes $\}$.

No overshooting of the vertices $a_{j}(j=1, \ldots, N+3)$ of a polygon $\Gamma$ was been observed in any numerical computations of generalized discrete minimal surfaces. Moreover, all computed discrete surfaces $u_{h}$ satisfy 
Plateau's boundary condition for $\Gamma$ in the sense that

$$
u_{h}\{\text { boundary nodes }\} \subset \Gamma
$$

and

$$
u_{h} \text { is order preserving on \{boundary nodes\}. }
$$

This fact justifies the use of our numerical approximation of the Marx-Shiffman function $\Theta$ for the computation of discrete minimal surfaces that are bounded by a polygon $\Gamma$ and might lead to the conjecture that $d$ and $\Theta$ coincide at their critical point set.

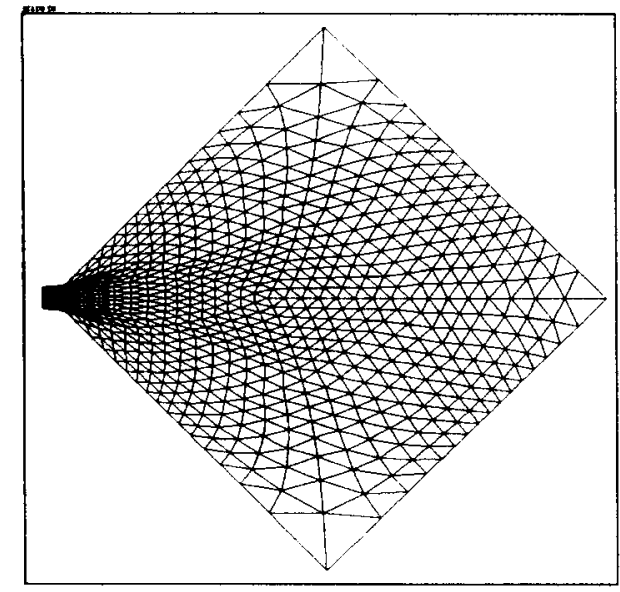

Figure 7: Initial Surface

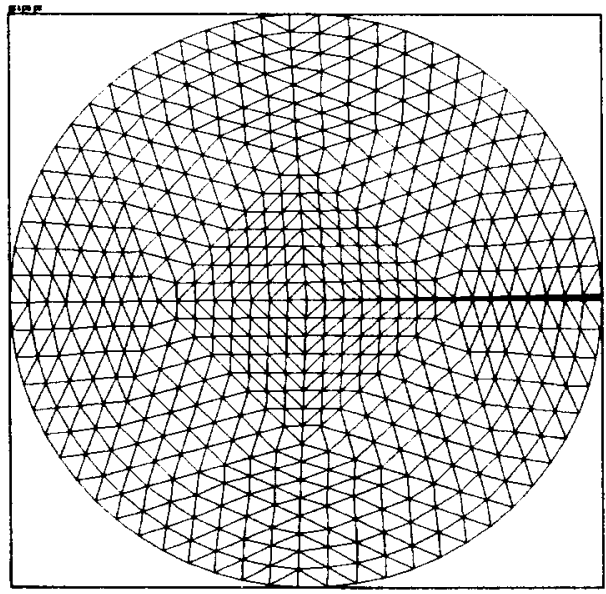

Figure 9: Final Triangulation

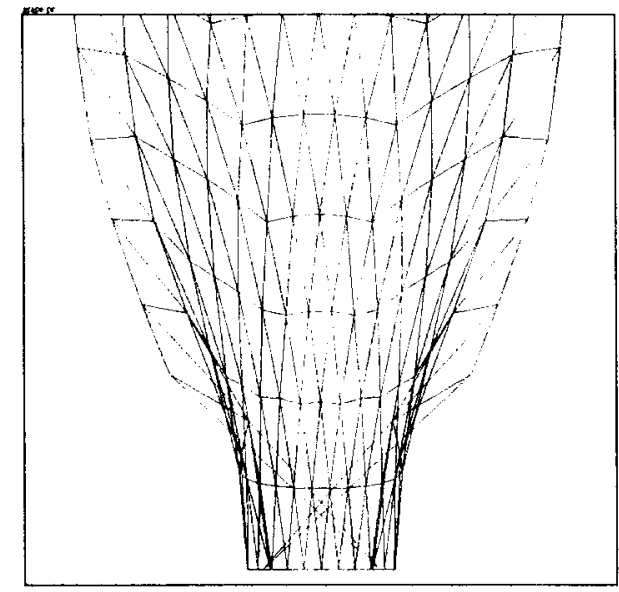

Figure 8: Zoom on $a_{1}+\Gamma_{1}$

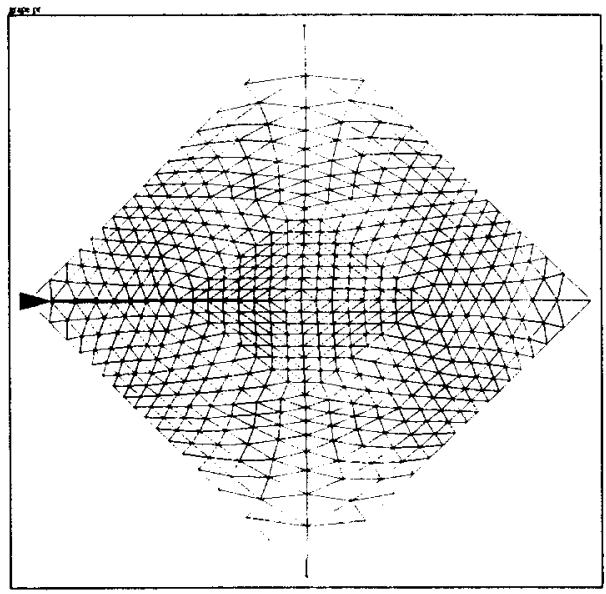

Figure 10: Discrete minimal surface

Example 5.3 Polygonal approximations of minimal surfaces bounded by Enneper contours 


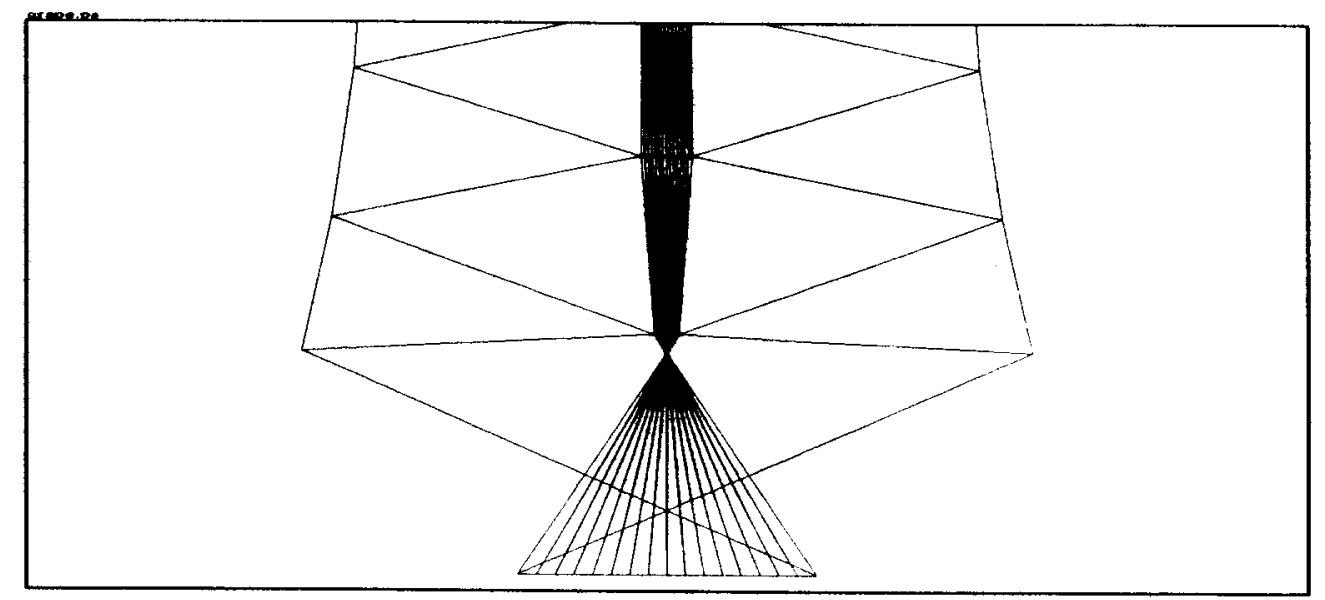

Figure 11: Discrete minimal surface, Zoom on $a_{1}+\Gamma_{1}$

The one-parameter family of Enneper contours is given by

$$
E_{r}(\varphi)=\left\{\begin{array}{r}
r \cos \varphi-\frac{1}{3} r^{3} \cos 3 \varphi \\
-r \sin \varphi-\frac{1}{3} r^{3} \sin 3 \varphi \\
r^{2} \cos 2 \varphi
\end{array} \quad \varphi \in[0,2 \pi]\right.
$$

Several authors investigated these contour-family and the corresponding minimal surfaces problem. The best general reference here is the famous book of Nitsche [17]. For a comprehensive numerical treatment we refer to [12, Section 5.4.2].

In order to obtain polygonal approximations of Enneper contours we proceeded as follows. For $n \in$ $\mathbb{N}, n \geq 4$ we subdivided the interval $[0,2 \pi]$ into $n$ parts

$$
\left[0, \varphi_{1}\right],\left[\varphi_{1}, \varphi_{2}\right], \ldots\left[\varphi_{n-1}, 2 \pi\right]
$$

where

$$
\varphi_{i}:=2 \pi \frac{i}{n} \quad 0 \leq i \leq n-1 .
$$

The polygon $\Gamma_{n}^{r}$ is defined by the $n=: N+3$ vertices $a_{i}:=E_{r}\left(\varphi_{i}\right)(0 \leq i \leq n-1)$.

In Figs. 12,13 we present the numerical results in the case $n=18$ and $r=1.2$. For numerical reasons we replaced the three-point condition (11) by more suitable ones. For the approximation we used triangulations containing 3816 triangles and 2197 nodes, where 576 nodes are boundary nodes. The energy of the first surface is 13.1547 , the discrete area has the value 13.0797 . The second and third surface both have the area 12.9218 , their energy has the value 13.1128 . The matrix $D^{2} \Theta_{h}\left(\kappa_{h}^{1}, 1.2\right)$ has one negative eigenvalue, the matrices $\left.D^{2} \Theta_{h}\left(\kappa_{h}^{i}\right), 1.2\right)(i=2,3)$ are positive definite. Therefore, these three discrete minimal surfaces might be approximations of an unstable critical point and two local minima of the area functional. 

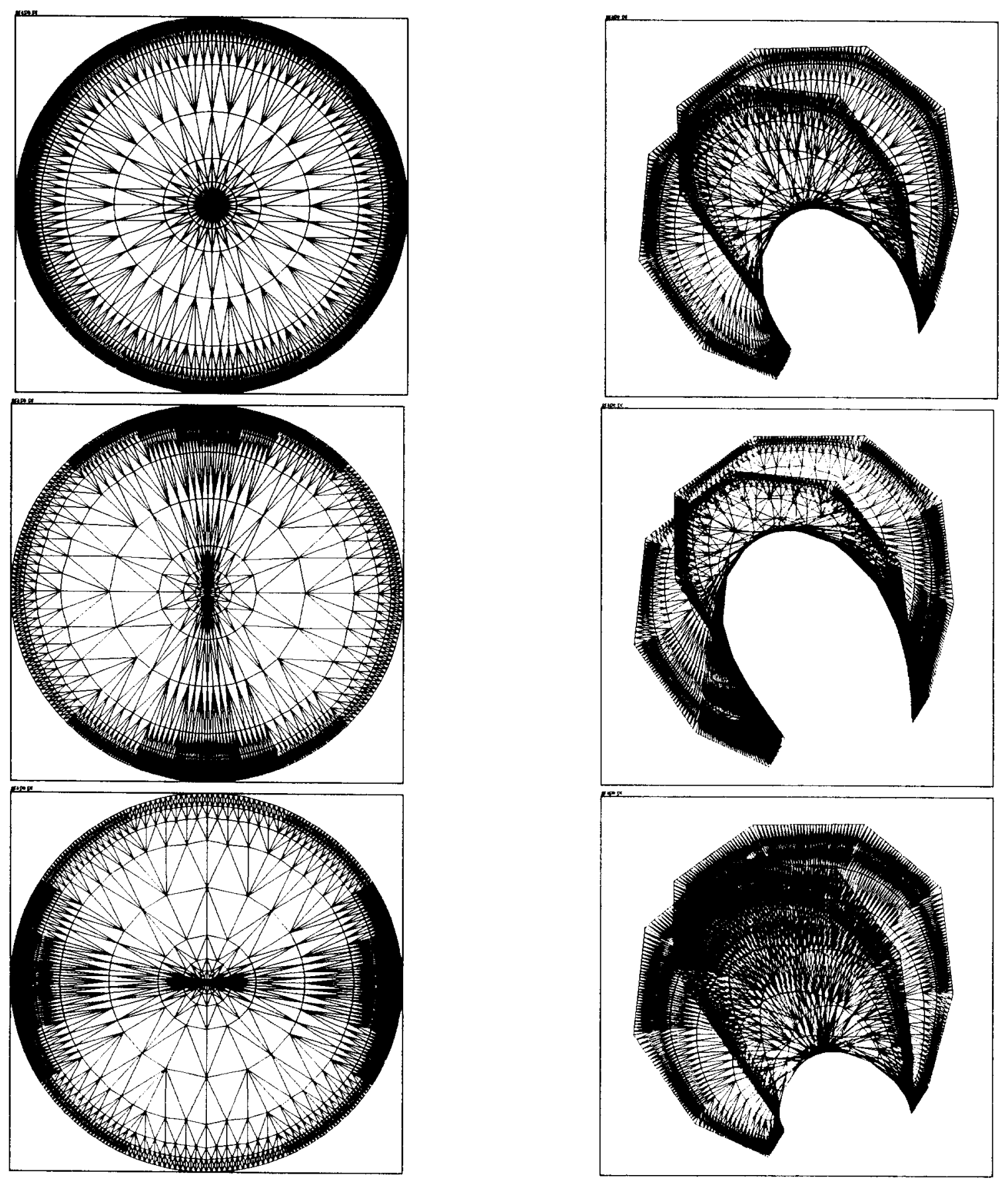

Figure 12: Triangulations, 3816 triangles

Figure 13: Corresponding minimal surfaces 


\section{References}

[1] H.W. Alt. Lineare Funktionalanalysis. Springer 1985

[2] R. Courant. Dirichlet's Principle. Interscience Publishers 1950

[3] G.Dziuk. An Algorithm for Evolutionary Surfaces. Numer. Math. 58, 603-611 (1991)

[4] G.Dziuk, J.E. Hutchinson On The Approximation Of Unstable Parametric Minimal Surfaces.

[5] E. Heinz. Über die analytische Abhängigkeit eines linearen elliptischen Randwertproblems von $\mathrm{Pa}$ rametern. Nachr. Akad. Wiss. Gottingen, 1-20 (1979)

[6] E. Heinz. Über eine Verallgemeinerung des Plateau'schen Problems. Manuscipta Math. 28, 81-88 (1979)

[7] E. Heinz. Ein mit der Theorie der Minimalflächen zusammenhängendes Variationsproblem. Nachr. Akad. Wiss. Göttingen, 25-35 (1980)

[8] E. Heinz. Zum Marx-Shiffmanschen Variationsproblem. J. Reine u. Angew. Math. 344, 196-200 (1983)

[9] E. Heinz. Minimalflächen mit polygonalem Rand. Math. Z. 183, 547-564 (1983)

[10] M. Hinze. Zur numerischen Behandlung des Marx-Shiffman-Randwertproblems. Diplomarbeit, Institut für Angew. Math., Universtät Bonn, 1989

[11] M. Hinze. On the Numerical Treatment of Quasiminimal Surfaces. Impact of Computing in Science and Engineering 5, 249-270 (1993)

[12] M. Hinze. On the Numerical Treatment of Minimal Surfaces with Polygonal Boundaries. Ph.D., Technical University Berlin, 1994

[13] J.E. Hutchinson. Computing Conformal Maps and Minimal Surfaces. Proc. Centr. Math. Anal., Canberra, 26 (1991)

[14] H. Jarausch Zur Numerischen Behandlung Von Parametrischen Minimalfläıhen Mit Finiten Elementen Dissertation, Ruhr Universität Bochum, Germany

[15] F. Lewerenz. Eine Bemerkung zu den Marx-Shiffman'schen Minimalvektoren bei Polygonen. Arch. Rat. Mech. Anal. 78 (1981)

[16] I. Marx. On the Classification of Unstable Minimal Surfaces with Polygonal Boundaries. Communications on Pure and Applied Mathematics, Vol. 8, 235-244 (1955)

[17] J.C.C Nitsche. Lectures on Minimal Surfaces Volume 1. Cambridge University Press 1989

[18] U. Pinkall, K. Polthier Computing Discrete Minimal Surfaces And Their Conjugates SFB 288 Preprint No. 49, Technical University Berlin, Germany

[19] F. Sauvigny. Ein Findeutigkeitssatz für Minimalfächen im $\mathbb{R}^{\prime \prime}$ mit polygonalem Rand. Journal f. d. Reine und Angew. Math. 358, 92-96 (1985)

[20] F. Sauvigny. On the Morse index of minimal surfaces in $\mathbb{R}^{p}$ with polygonal boundaries. Manuscripta math. 53, 167-197(1985) 
[21] T. Tsuchija. On Two Methods For Approximating Minimal Surfaces In Parametric Form. Math. Comp. 46, 512-529 (1986)

[22] T. Tsuchija. Discrete Solution of the Plateau Problem and Its Convergence. Math. Comp. 49, 157-165 (1987)

[23] T. Tsuchija. A Note On Discrete Solutions Of the Plateau Problem. Math. Comp. 54, 131-1.38 (1990)

[24] O. Wohlrab. Zur numerischen Behandlung von parametrischen Minimalflächen mit halbfreien Rändern. Preprint No. 739, SFB 72 Universität Bonn 
